\title{
A novel experimental approach for studying spontaneous imbibition processes with alkaline solutions
}

\author{
T. Chevalier ${ }^{1}, J$. Labaume $^{1}, A$. Delbos $^{1}, T$. Clemens $^{2}, V . M$. Waeger $^{2}, B$. Bourbiaux $^{1}$, and $M$. Fleury $^{1, *}$ \\ ${ }^{1}$ IFPEN, Rueil-Malmaison, France \\ ${ }^{2} \mathrm{OMV}$, Vienna, Austria
}

\begin{abstract}
Spontaneous imbibition processes can play an important role in oil production. It can be enhanced or influenced by wettability changes generated by properly designed chemicals or by the natural surfactants resulting from reactive crude oils in the presence of alkaline solutions. The reaction of basic salts with some components of oil can, indeed, lead to the formation of natural soaps that reduces the interfacial tension between oil and brine. The latter scenario is studied herein on samples and oil from the St Ulrich oil field in the Vienna basin. To that end, spontaneous imbibition experiments were performed with two brines differing by the absence or presence of alkali. We first present a general novel technique to monitor saturation changes on small rock samples for the purpose of assessing the efficiency of a given recovery process. Samples of only $15 \mathrm{~mm}$ in diameter and $20 \mathrm{~mm}$ in length and set at irreducible saturation were fully immersed in the solution of interest, and the evolution of the samples' saturation with time was monitored thanks to a dedicated NMR technique involving the quantification of the sole oil phase present within the sample. A fully-3D imbibition configuration was adopted, involving counter-current flows through all faces of the sample. The experimental method is fast for two reasons: (i) the kinetics of capillary imbibition process is proportional to the square of sample size, i.e. very rapid if accurate measurements can be acquired on tiny samples, (ii) the present 3D situation also involves faster kinetics than the 1D configuration often used. The NMR technique was crucial to achieve such conditions that cannot be satisfied with conventional volumetric methods. The kinetics of oil desaturation during spontaneous imbibition is interpreted with the help of an analytical 3D diffusion model. For the alkaline solution, the diffusion coefficient is reduced by a factor of only two compared to the non-alkaline brine, although the interfacial tension between the oil and the imbibing solution is reduced by a factor of 10 . Hence, a wettability change to a more water wet state has to be assumed when the alkaline solution replaces the non-alkaline solution in the imbibition process. However, no significant impact on the final saturation was observed.
\end{abstract}

\section{Introduction}

It is widely recognized that reservoir wettability affects the relative distribution of fluids within a porous medium, which in turn strongly affects the displacement behavior, capillary pressure and relative permeability characteristics and consequently, the production of hydrocarbons from petroleum reservoirs.

Wettability change of rock samples can be achieved using injection of chemicals ${ }^{1,2}$ or low salinity brines ${ }^{3}$. The method studied in this report for the St Ulrich rock samples consists of the injection of alkaline brine ${ }^{4}$. The wettability change in this case is reported to be a combination of two phenomena ${ }^{5,6}$. First the brine composition changes, thus involving mechanisms similar to the ones invoked for low-salinity flooding effects. The second mechanism stems from the reaction of acid and/or basic oil components with alkali leading to the formation of in-situ surfactants (soaps) that can alter the wettability of the rock.

The objective herein is to study the wettability change of reservoir rock samples under alkaline conditions via spontaneous imbibition experiments. Indeed, when wettability is modified, the spontaneous imbibition capillary pressure curve is a sensitive indicator. To do so, reservoir samples are first aged with reservoir oil at irreducible saturation, and the imbibition of a reference injection brine or of an alkaline brine is measured. It is expected that the difference between these two experiments will give the answer. Since imbibition experiments are well known to be time consuming when using plugs of standard diameter $(40 \mathrm{~mm})$, it was decided to perform these experiments with smaller rock samples (15 mm dia) using low field NMR to monitor the oil saturation. We present an original method to measure oil saturation during imbibition, in which we acquire data at regular time intervals varying from 1 hour up to 1 day. Finally we analyze the recovery kinetics with an approach based on the equivalence of imbibition to a diffusion

* corresponding author: marc.fleury@ifpen.fr 
mechanism. Capillary diffusion coefficients are determined using an analytical formulation for both imbibition types (i.e. for the injection and the alkaline brines). In this paper, we also focus on the interpretation of this coefficient and the validation of some assumptions leading to the capillary diffusion approach.

\section{Material and methods}

\subsection{Samples and fluids}

Brine recombination: Two brine compositions were considered in relation to the field operating conditions: the reservoir connate brine composition and the composition of the brine used for field waterflooding (Table 1). The latter brine is denoted as the injection brine in the following.

Three synthetic brines were reconstituted on the basis of these two compositions as explained hereafter. The reservoir connate brine composition was only used to restore the initial saturation of rock samples, that is oil in the presence of irreducible brine. The injection brine composition was used to perform the reference imbibition experiment. It is similar to the connate brine but with a smaller total dissolved salts. Alkaline imbibition experiments were performed with the injection brine composition in which we removed the initials divalent ions and added sodium carbonate at a concentration of 15 $\mathrm{g} / \mathrm{l}$ (divalent ions were removed to avoid precipitation). Note that to remove the signal of the water from the NMR spectrum, $\mathrm{D}_{2} \mathrm{O}$ is used for the brine preparation instead of $\mathrm{H}_{2} \mathrm{O}$. The brines compositions are summarized in Table 1 .

Table 1: Composition of the different brines used in the experiments ; connate brine at Swi, injection brine for imbibition experiments on samples $1 \mathrm{a}$ and $2 \mathrm{a}$, and alkaline brine for experiments on samples $1 b$ and $2 b$

\begin{tabular}{|l|l|l|l|}
\hline \multirow{2}{*}{$\begin{array}{l}\text { Synthetic } \\
\text { brine } \\
\text { composition }\end{array}$} & \multicolumn{3}{|l|}{ Concentration $(\mathrm{g} / \mathbf{L})$} \\
\cline { 2 - 4 } & Connate brine & $\begin{array}{l}\text { Injection } \\
\text { brine }\end{array}$ & $\begin{array}{l}\text { Alkaline } \\
\text { brine }\end{array}$ \\
\hline $\mathrm{NaCl}$ & 15 & 10.5 & 10.5 \\
\hline $\mathrm{KCl}$ & 0.145 & 0.09 & 0.09 \\
\hline $\mathrm{MgCl} 2$ & 0.52 & 0.28 & \\
\hline $\mathrm{CaCl} 2$ & 0.5 & 0.34 & \\
\hline $\mathrm{Na} 2 \mathrm{CO} 3$ & & & 15 \\
\hline
\end{tabular}

Oil and interfacial tensions: Crude oil samples were collected from the producing well Saint Ulrich 65. The samples were analysed in terms of oil-water interfacial tension (IFT) to verify that no additive was present ${ }^{2}$ in the crude oil. Its water content was also measured using the Karl Fischer method, and checked to be low. API gravity, viscosity and density of that crude oil were determined to be respectively $31^{\circ} \mathrm{API}, 8.2 \pm 0.1 \mathrm{cP}$ (at $100 \mathrm{~s}^{-1}$ and $40^{\circ} \mathrm{C}$ ) and $0.854 \pm 0,001 \mathrm{~g} / \mathrm{cm}^{3}$ (at $40^{\circ} \mathrm{C}$ ). The oil was then filtered at the reservoir temperature to avoid any plugging during the injection in porous media. The interfacial tension (IFT) was measured using the Wilhelmy plate method at $40^{\circ} \mathrm{C}$ (Figure 1). For the oil-alkaline brine system, the IFT is much lower $(0.8 \mathrm{mN} / \mathrm{m})$ compared to the oil-injection brine system $(8 \mathrm{mN} / \mathrm{m})$. This is an expected effect since the presence of alkali in the brine may react with some components of the oil to form natural soaps.

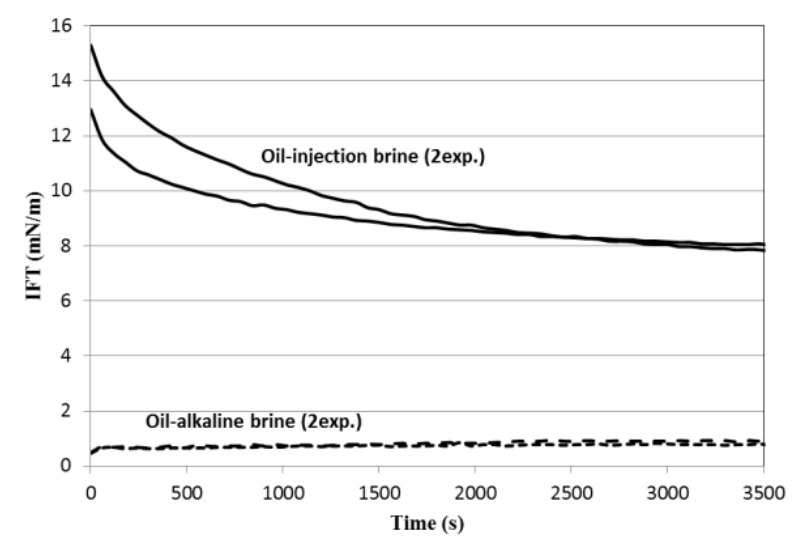

Fig. 1. Interfacial tension, determined by Wilhelmy plate method at $40^{\circ} \mathrm{C}$, between oil and the injection brine (full lines) and alkaline brine (dashed lines). Experiments were repeated twice.

Plug selection and restoration: Small cylindrical samples ("plugs") have been drilled from turbiditic Flysch sandstones of Eocene age. The Flysch in Austria constitutes a fractured siliciclastic basement reservoir. Flow paths are mainly provided by fractures whereas some storage capacity is present in the matrix. The samples itself can be described as fine-medium-grained, moderately well sorted sandstones. Mineralogical composition is dominated by quartz with minor feldspar and low volumes of glauconite. The samples chosen for imbibition have not been used for porosity-permeability measurements. Matrix porosity and permeability from a nearby drilled plug are respectively $8.3 \%$ and $0.3 \mathrm{mD}$. Reservoir properties of the matrix are mainly deteriorated by compaction and quartz cementation.

To select samples as homogeneous as possible, plugs were scanned as received. Four sister plugs among the most homogeneous ones were selected for the study.

Small cylindrical samples were cored through these plugs, dried and saturated with the connate brine. They were then set at Swi using centrifuge. Once at Swi, they were aged for 3 weeks at reservoir temperature. During that aging period, cores were flooded every week with one pore volume of dead oil. This aging time is considered adequate to restore the in-situ wettability.

\subsection{NMR experimental set-up}

The experimental set-up described in detail elsewhere ${ }^{7}$ is briefly summarized here. The spontaneous imbibition is done in a glass tube of outer diameter $18 \mathrm{~mm}$ closed with a plastic cap to prevent evaporation of the brine during the process as illustrated on Figure 2. Imbibition cells remain in an oven at $40^{\circ} \mathrm{C}$ except during the measurements performed in the NMR spectrometer at $30^{\circ} \mathrm{C}$ (a MARAN 23.7 $\mathrm{MHz}$ from Oxford Instruments equipped with a 18 
mm diameter probe). The experiment lasted two weeks and the interval between two measurements varied between $30 \mathrm{~min}$ at the beginning of the process and several hours at the end. This frequency of measurement is designed to capture the rapid evolution of the saturation during the first day of imbibition, followed by a much slower kinetics afterwards.

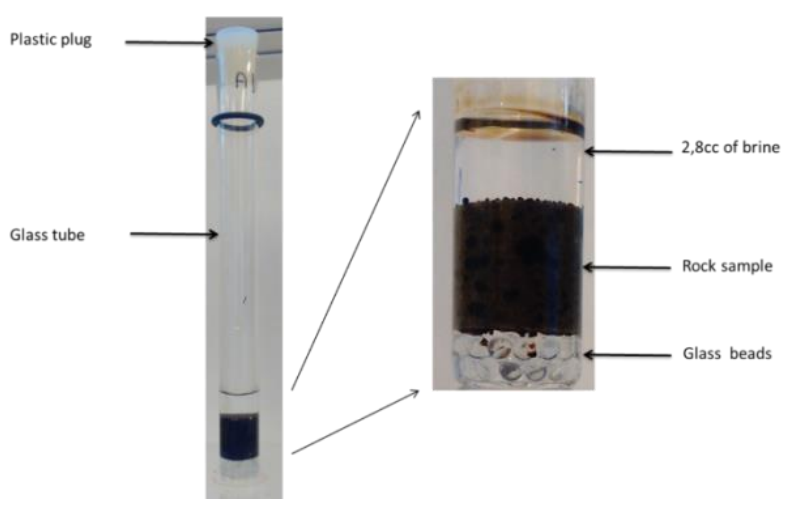

Fig. 2. Imbibition tube placed in the NMR spectrometer (outer diameter $18 \mathrm{~mm}$ ).

In these experiments, the aqueous solution imbibes through all the faces of the sample. As spontaneous imbibition process is known to have a slow kinetics, it is advantageous to work with small samples: the NMR technique is crucial, making possible the detection of some hundreds of micro-liter in the sample with reasonable accuracy (Table 2). During the spontaneous imbibition, oil is expelled out of the sample and segregates in the $\mathrm{D}_{2} \mathrm{O}$ brine that surrounds the sample, but above the sensitive volume investigated by the NMR probe. By using deuterium instead of hydrogen in the brine, all the measured NMR signal emanates from the oil held in the sample and we can obtain the oil saturation without the need for separating oil and water in $T_{2}$ distributions. However, $\mathrm{T}_{2}$ distributions were needed to eliminate a small contribution of oil droplets temporarily sticking at the surface of the sample (Figure 2); a cut-off above $100 \mathrm{~ms}$ characterizing these droplets has been found adequate and all $\mathrm{T}_{2}$ components above $100 \mathrm{~ms}$ have been removed to calculate the oil saturation.
Darcy's law governing two phase flow in porous media can be written in terms of a unique convection-diffusion equation $^{8}$. The spontaneous imbibition of very small samples as the ones used in this study involves negligible convection and buoyancy effects, which yields the following equation:

$$
\begin{gathered}
\Phi \frac{\partial S_{w}}{\partial t}=-\nabla\left[K \frac{K_{w} K_{o}}{K_{t}} \nabla P_{c}\left(S_{w}\right)\right] \text { with } K_{o}= \\
\frac{k_{r o}}{\mu_{o}}, K_{w}=\frac{k_{r w}}{\mu_{w}}, \\
K_{t}=K_{o}+K_{w}
\end{gathered}
$$

in which $\mathrm{K}$ is the single-phase permeability, $\mathrm{S}_{\mathrm{w}}$ is the water saturation, $\mathrm{K}_{\mathrm{w}, \mathrm{o}}$ are the relative mobility for oil (o) and water $(\mathrm{w})$ and $\mu_{\mathrm{o}, \mathrm{w}}$ is the viscosity of oil and water respectively. Porosity is assumed constant. Introducing the imbibition capillary pressure curve $\operatorname{Pc}\left(\mathrm{S}_{\mathrm{w}}\right)$, the above equation can be rewritten as a diffusion equation:

$$
\begin{aligned}
\frac{\partial S_{w}}{\partial t} & =D_{c} \Delta S_{w} \quad \text { with } \\
D_{C} & =\frac{1}{\phi} K \frac{K_{w} K_{o}}{K_{t}} \frac{\partial P_{c}}{\partial S_{w}}
\end{aligned}
$$

where $D_{c}$ is called a capillary diffusion coefficient to emphasize that capillary forces are presumably the driving forces in the present study. When $\mathrm{D}_{\mathrm{c}}$ is constant, the solution of the diffusion equation 2 in three dimensions corresponding to the experimental situation (a cylinder) is well known ${ }^{9}$. After normalizing, the oil saturation So* is given by:

$$
\begin{gathered}
S_{o}^{*}=C_{p s} C_{c y l} \quad \text { with } \quad S_{o}^{*}=\frac{S_{o}-S_{o f}}{S_{o i}-S_{o f}} \\
C_{p s}=\sum_{n=0}^{\infty} \frac{8}{(2 n+1)^{2} \pi^{2}} \exp \left(-D_{c}(2 n+1)^{2} \pi^{2} \frac{t}{4 l^{2}}\right) \\
C_{c y l}=\sum_{n=1}^{\infty} \frac{4}{r^{2} q_{n}^{2}} \exp \left(-D_{c} q_{n}^{2} t\right)
\end{gathered}
$$

\begin{tabular}{|c|c|c|c|c|c|c|c|c|c|c|c|}
\hline \multicolumn{5}{|c|}{ Sample } & \multirow[t]{2}{*}{ Brine type } & \multicolumn{2}{|c|}{ Initial } & \multicolumn{3}{|c|}{ Recovery } & \multirow[t]{2}{*}{ Dc } \\
\hline $\mathrm{n}^{\circ}$ & $\mathrm{H}$ & Diam. & $\Phi$ & $\mathrm{V}_{\mathrm{p}}$ & & $\mathrm{V}_{\mathrm{oi}}$ & $\mathrm{S}_{\mathrm{wi}}$ & $\Delta \mathrm{V}_{\mathrm{o}}$ & $\underset{\mathrm{oi}}{\Delta \mathrm{V}_{\mathrm{o}} / \mathrm{V}}$ & Sor & \\
\hline & {$[\mathrm{mm}]$} & {$[\mathrm{mm}]$} & {$[\%]$} & {$[\mu \mathrm{L}]$} & & {$[\mu \mathrm{L}]$} & & {$[\mu \mathrm{L}]$} & & & {$\left[\mathrm{cm}^{2} / \mathrm{s}\right]$} \\
\hline $1 \mathrm{a}$ & 18.3 & 14.7 & 10.0 & 310 & Injection & 277 & $11 \%$ & 123 & $44 \%$ & $50 \%$ & $1.210^{-6}$ \\
\hline $1 \mathrm{~b}$ & 18.1 & 14.7 & 10.0 & 307 & Alkaline & 265 & $14 \%$ & 117 & $44 \%$ & $48 \%$ & $6.010^{-7}$ \\
\hline $2 a$ & 17.1 & 14.7 & 10.2 & 295 & Injection & 246 & $17 \%$ & 109 & $44 \%$ & $46 \%$ & $9.810^{-7}$ \\
\hline $2 b$ & 18.2 & 14.7 & 9.1 & 282 & Alkaline & 261 & $8 \%$ & 114 & $44 \%$ & $52 \%$ & $6.610^{-7}$ \\
\hline
\end{tabular}

\section{Theory}

Table 2: Samples characteristics (sizes, porosity and porous volume), brine type, initial and final saturation. Capillary diffusion coefficient (Dc) from diffusion analysis (see text and Figure 6). 
where $\mathrm{q}_{\mathrm{n}}$ are the positive roots of the equation $J_{0}\left(r q_{n}\right)=0, J_{0}$ being the first type Bessel function of order zero, $r$ is the radius of the cylinder and $2 l$ its length. $\mathrm{S}_{\mathrm{oi}}$ and $\mathrm{S}_{\mathrm{of}}$ are respectively the initial and final oil saturation at the end of spontaneous imbibition. The boundary condition associated with the above solution is a constant "saturation" outside the sample $\left(\mathrm{S}_{\mathrm{w}}=1\right)$. This model has been used successfully in the past ${ }^{10}$ to analyze deuterium exchange experiments solely governed by molecular diffusion of water within a cylindrical porous medium in the context of tight very low permeability porous media.

For the case of spontaneous experiments, the constant diffusion coefficient Dc for solving equation 2 is a significant simplification and is worth examining further. This coefficient depends obviously on saturation and is therefore not spatially constant within the sample. For this purpose and as in a previous work $^{11}$, we calculated Dc taking standard $\mathrm{Kr}$ curves and an empirical imbibition Pc curve. The latter took into account the actual pore size distribution of the porous medium under consideration, as inferred from a mercury injection curve. The coefficient Dc is nearly constant within a large saturation range (Figure 3, $\mathrm{Sw}^{*} \in[0.2-0.8]$ ) and decreases sharply outside this range (the Dc value close to experimental ones is coincidental). Hence when fitting the data, we can expect some deviations from the data points in addition to experimental uncertainties. As an example, we simulated an oil desaturation curve for a coefficient De varying from 1 up to $3 \times 10^{-10} \mathrm{~m}^{2} / \mathrm{s}$ (Figure 4); in this case, one can expect deviations from the best fit by about $\pm 10 \%$.

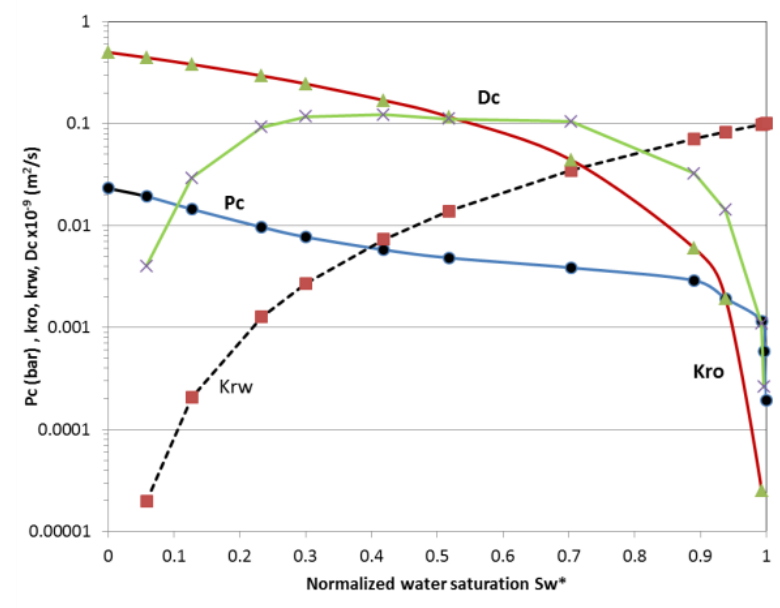

Fig. 3. Calculation of the capillary diffusion coefficient Dc using equation 2 taking typical $\mathrm{Kr}$ curves and an empirical imbibition capillary pressure curve.

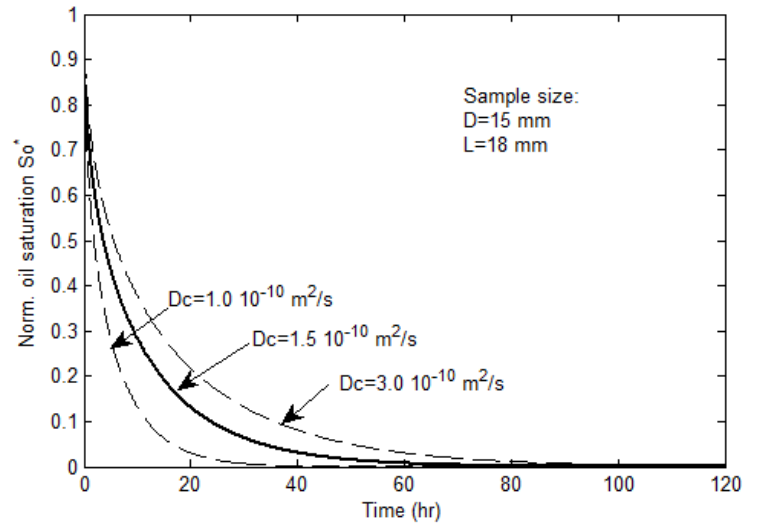

Fig. 4. Sensitivity of the oil saturation decay curve when the diffusion coefficient is varied within a factor of 3 using equation 3.

\section{Results}

All the measured desaturation curves are shown in Figure 5 . The initial and final water saturation varies respectively from $8 \%$ to $17 \%$ and $46 \%$ to $52 \%$, the variation of saturation being however similar for all samples $(44 \%$, Table 2). Data points were also collected after $150 \mathrm{~h}$ (up to $350 \mathrm{~h}$, not shown) allowing to observe a very stable saturation. At the end of the tests, samples were removed from the glass tubes, oil droplets sticking on the surface carefully wiped off, and oil saturation measured again. Hence we could verify that the cut-off approach mentioned above gives a reasonable estimate of the true saturation inside the sample; indeed the oil saturation inside (with droplets and signal correction) and outside (without droplets) the tube could differ by at most 4 saturation units $(4 \%)$, while the contribution of the components above $100 \mathrm{~ms}$ can be much larger.

A careful examination of the curves reveals some differences when comparing data points $1 \mathrm{a}$ and $1 \mathrm{~b}$, and $2 \mathrm{a}$ and $2 \mathrm{~b}$ : oil flows out faster from the samples without alkaline salt (samples 1a and 2a) than from the ones with alkali. Such behaviour will become more obvious when fitting the data with the capillary diffusion model.

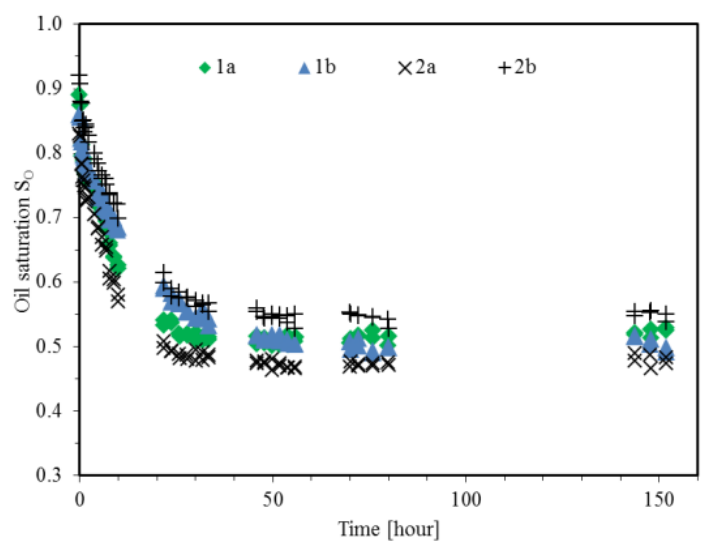

Fig. 5. Desaturation curves obtained for all samples. Imbibition experiments on samples $1 \mathrm{a}$ and $2 \mathrm{a}$ was performed with the injection brine, and $1 \mathrm{~b}$ and $2 \mathrm{~b}$ with the alkaline brine. 
We fitted the 4 data curves with the diffusion model (equation 3 ) by normalizing the oil saturation between 1 and 0 (Figure 6, the asymptotic oil saturation at infinite time was chosen as the value measured outside the tube and is indicated as a horizontal line). First, we note that the experimental kinetic trend is consistent with a diffusion approach of the imbibition process. At first glance, this was not obvious because all the coefficients $\mathrm{K}_{\mathrm{w}}, \mathrm{K}_{\mathrm{o}}$ and $\mathrm{K}_{\mathrm{t}}$ depend on saturation, yielding potentially a non-unique coefficient Dc. Second the capillary diffusion coefficients Dc can be split into two groups: the ones referring to imbibition with alkaline brine and the ones referring to imbibition without alkali. Dc values without alkali are between 1.5 and 2 times larger than Dc values with alkaline brine. That difference reflects qualitative observations that oil desaturation during the first 50 hours is slower with the alkaline brine than without alkaline. However, and despite these differences, the ultimate recovery reached after about 3 days of spontaneous imbibition, is similar whatever the brine type as already pointed out before.
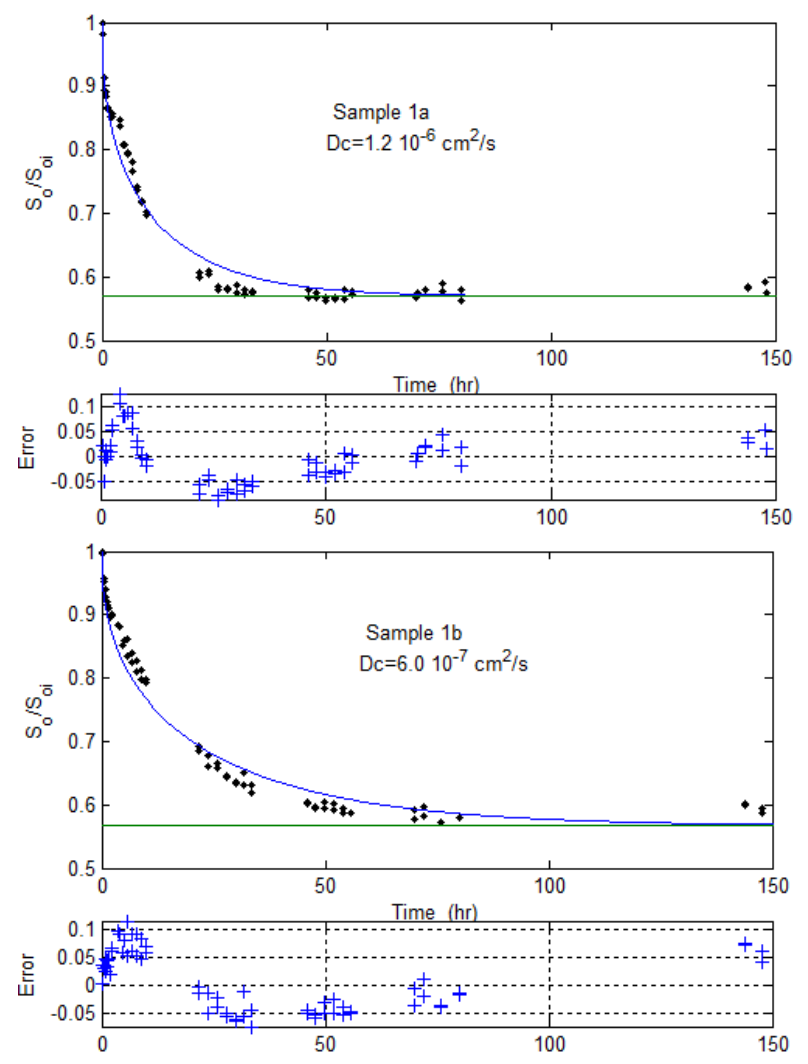
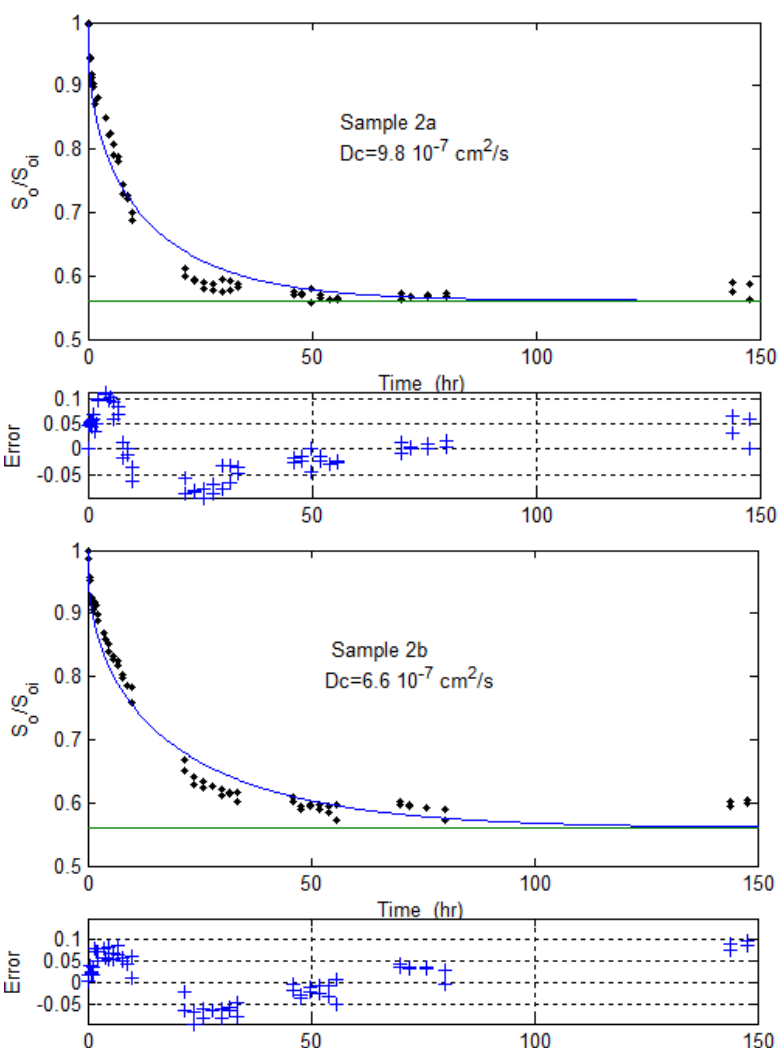

Fig. 6. Desaturation curves fitted with the capillary diffusion model, equation 2.

\section{Discussion}

Experimental data points are reasonably well fitted by a constant capillary diffusion model, indicating that the simplified equation can describe the kinetic of the process; the error is indeed mostly comprised within $\pm 5 \%$ (Figure 6). We discuss below three aspects: the size effect on the experimental duration, the wettability modification by the alkaline solution, and the comparison between other processes occurring simultaneously during the imbibition process.

Typical experimental durations are comprised between 50 and 100 hours (Figure 6) for the present sample sizes (D 15mm, L 18mm). By comparison with experiments made on standard plug size (D 40mm, $\mathrm{L} \sim 60 \mathrm{~mm}$ ), the duration will be reduced by the square of the characteristic length Lc (as any diffusion process), where Lc can have several expressions depending on the author: $L c=L D / 2 \sqrt{2 L^{2}+D^{2}} 12$ or $L c=L D /(2 D+4 L)$ 13,11 . With both formula, the experimental time is reduced by a factor of 8 . Comparing 1D (opposite face open) to 3D imbibition experiments also reduces the experimental time. We similarly evaluated the experimental durations in $3 \mathrm{D}$ with equations 3 to 5 and found a similar factor. Hence, the experimental set-up used in this study is quite efficient for screening imbibition mechanisms influenced or not by chemicals.

One has still to explain the kinetic difference between alkaline brine and injection brine (Table 2, Dc column). To that end, one has to consider the modification of surface and interfacial properties of the rock-fluid system 
in the presence of alkali. If one assumes a fixed wettability of the rock-fluid system, then the decrease of water-oil IFT in the presence of alkali reduces the imbibition kinetics in the same ratio because the intensity of the driving capillary forces is proportional to the capillary pressure gradient (equation 2) and therefore to the interfacial tension. Herein, the IFT is reduced by a factor close to 10 (Figure 1), however the imbibition kinetics as quantified by the capillary diffusion coefficient Dc is reduced by a factor not exceeding 2 . To explain that apparent discrepancy, one has to assume that a rock wettability change was induced under alkaline conditions $^{2}$. That wettability alteration impacts the intensity of capillary pressure but not the residual oil after imbibition (Figure 7). Note that this comparison is valid because samples have nearly the same porosity and permeability.

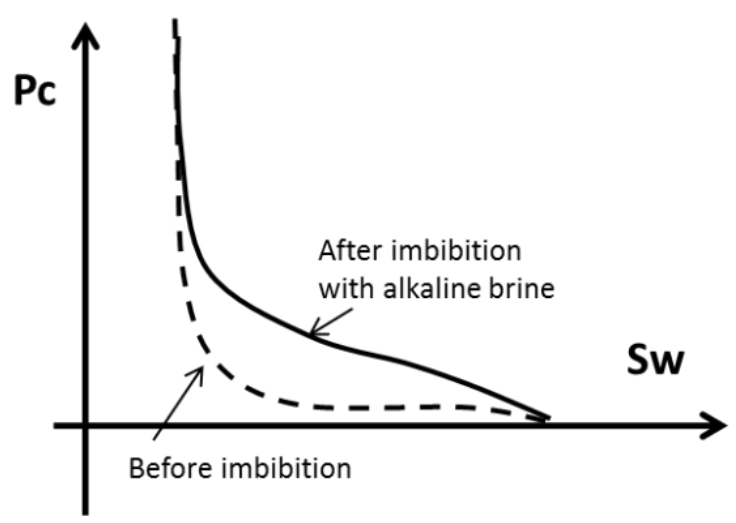

Fig. 7: Schematic of the wettability change to a more water-wet situation after imbibition with alkaline brine.

Finally, we can also discuss the various time scales of processes occurring simultaneously during the spontaneous imbibition mechanism. First, the effective molecular diffusion De of the alkaline brine solution into injection brine; it can be estimated using Archie relationships as De $\Phi S w(m=n=2)$. Hence De will be initially 100 times smaller than the bulk molecular diffusion coefficient of water, i.e. of the order of $4 \times 10^{-11}$ $\mathrm{m}^{2} / \mathrm{s}$, much smaller than the measured coefficient Dc. Second, the change of interfacial tension as the alkaline solution imbibes can be considered as instantaneous, as shown by measurements (Figure 1). Third, the time scale of the change of wettability must also be fast compared to the observed imbibition process.

\section{Conclusion}

During this study, spontaneous imbibition experiments have been performed to evaluate the ability of an alkaline brine to increase the oil recovery. The use of small samples $(D=15 \mathrm{~mm}$ and $\mathrm{L}=18 \mathrm{~mm})$ together with $\mathrm{NMR}$ volume measurements provided a significant speed-up of the experimental duration without loss of accuracy. Oil saturation has been measured as a function of time during the imbibition process occurring across all the surfaces of the sample. Using a simplified equation in 3 dimensions, a capillary diffusion coefficient Dc is calculated from the experiments to quantify the dynamics of the process. At the end of the experiments, the oil saturation is the same for the two sets of samples. However, the dynamics of the process is not the same. In the case of an alkaline brine, the oil production is slower (Dc is smaller) compared to the reference experiments performed with injection brine. However, since the interfacial tension is divided by about 10 in the presence of the alkaline solution and Dc only by a factor of 2 at most, we conclude that a wettability change occurred simultaneously during the imbibition process governed by capillary forces.

\section{References}

1. Chabert M, Morvan M, Tabary R. Fractured Carbonates: A Methodology to Evaluate Surfactant Performances. In: SPE Improved Oil Recovery Symposium., 2010.

2. Hirasaki G, Zhang DL. Surface Chemistry of Oil Recovery From Fractured, Oil-Wet, Carbonate Formations. SPE Journal. 2004;9(02):151-162.

3. Morrow N, Buckley J. Improved Oil Recovery by Low-Salinity Waterflooding. Journal of Petroleum Technology. 2013;63(05):106-112.

4. Cooke CE, Williams RE, Kolodzie PA. Oil Recovery by Alkaline Waterflooding. Journal of Petroleum Technology. 1974;26(12):1365-1374.

5. Ehrlich R, Hasiba HH, Raimondi P. Alkaline Waterflooding for Wettability Alteration-Evaluating a Potential Field Application. Journal of Petroleum Technology. 1974;26(12):1335-1343.

6. Campbell TC, Krumrine PH. Laboratory Studies On Alkaline Waterflooding. In: SPE Annual Technical Conference and Exhibition., 1979.

7. Chevalier T, Labaume J, Gautier S, Chevallier E, Chabert M. A Novel Experimental Approach for Accurate Evaluation of Chemical EOR Processes in Tight Reservoir Rocks. In: SPE Improved Oil Recovery Conference., 2018.

8. Chavent G, Jaffre J. Mathematical models and finite elements for reservoir simulation: Single phase, multiphase and multicomponent flows through porous media. Studies in mathematics and its applications. vol. 17. Amsterdam [etc.]: NorthHolland, 1986.

9. Crank J. The mathematics of diffusion (2nd edition). Oxford: Clarendon Press, 1976.

10. Berne P, Bachaud P, Fleury M. Diffusion Properties of Carbonated Caprocks from the Paris Basin. Oil \& Gas Science and Technology - Revue de l'Institut Français du Pétrole. 2009;65(3):473-484.

11. Stoll M, Hofman J, Ligthelm DJ, Faber MJ, van den Hoek P. Toward Field-Scale Wettability Modification-The Limitations of Diffusive Transport. SPE Reservoir Evaluation \& Engineering. 2013;11(03):633-640.

12. Ma S, Zhang $X$, Morrow N. Influence of Fluid Viscosity on Mass Transfer Between Rock Matrix and Fractures: SPE-99-07-02-PA. J. Can. Pet. Technol. 1999;38(7):25-30. 
13. Bourbiaux B, Fourno A, Nguyen Q-L, Norrant F, Robin M, Rosenberg E, Argillier J-F. Experimental and Numerical Assessment of Chemical Enhanced Oil Recovery in Oil-Wet Naturally Fractured Reservoirs. SPE Journal. 2016;21(03):706-719. 